\title{
The Atomic Regular Polyhedron Electronic Shell
}

\author{
Zilong Kong \\ Wufangfa Computer Technology Service Department, Guangzhou, China \\ Email: kongzl@oceanmining.org
}

Received June 30, 2013; revised August 2, 2013; accepted September 3, 2013

Copyright (C) 2013 Zilong Kong. This is an open access article distributed under the Creative Commons Attribution License, which permits unrestricted use, distribution, and reproduction in any medium, provided the original work is properly cited.

\begin{abstract}
The periodic table of elements is arranged based on a series of regular polyhedron. The stability of inert gas atoms can be explained by the distribution of electrons, as well as their motion and magnetic force structure. A magnetic force regular octahedron is proposed. It is a unique configuration that best satisfies the convergence of electrons moving in the same direction within regular polyhedra. In the case of an electrostatic force crust, the formal electron spin accounts for the crusts intrinsic magnetic moment exceeding the speed of light. If one is to consider that the electron has a magnetic outer layer and an electrostatic inner layer, then the question can be solved and abovementioned inference can provide the basis for magnetic force and momentum for the regular octahedron model. The electron periphery has twenty-petal adsorptive substances; the existence of adsorptive substance causes the magnetic force greater than the electrostatic force. Each electronic shell in the regular polyhedron is in accordance with the electron configuration of periodic table of elements; the kinetic track of each electron is a surface of regular polyhedron. The magnetic properties of iron, cobalt, and nickel can be explained by the regular dodecahedron electronic shell of an atom. The electron orbit converged from reverse direction can explain diamond. The adsorptive substances found in atomic nuclei and electrons are defined as magnetic particles called magnetons. The thermodynamic magneton theory can be better explained when it is analyzed using principles of thermodynamics, superconductivity, viscosity, and even in the creation of glass. The structure of the light is a helical line.
\end{abstract}

Keywords: Atomic Structure; Regular Polyhedron; Electronic Shell; Periodic Table of Elements; Light; Diamond

\section{Introduction}

The study of quantum mechanics entails quantifying atom from energy and momentum, wave properties of particle and other aspects, and various electron configurations. Based on the structure of hydrogen atom, a single electron system is initially developed. The present method is still confined to the energy level, as in a unitary approach. Solid and liquid do not have characteristic spectrum, and they lacked an item of information in energy difference. This article regards the inert gases atom as the initiation point for multiple electron system. The magnetic force structure, in which the electrons interact, is analyzed for the formation of electron configuration, using the simple geometric model, which provides the obvious details.

The octet rule [1] describes the most appearance probability of electrons of stable inert gases atoms configuration situated at the corners of the cube, and this may regard as which indicates that the electrons are close to resting. Now this article discusses the state at which electrons are moving: if one is to disregard that each electron motion must be around the atomic nucleus, changing its motion to a circle around the line that connects the nucleus with eight vertices of cube, then a configuration of eight electrons with stable motion is gained. Its symmetry is unique in the magnetic force structure.

If the electron has an electrostatic force crust, the formal electron spin accounts for the crusts intrinsic magnetic moment exceeding the speed of light, which was found by Wolfgang Pauli.

Compton wavelength $\lambda_{C}$ of electron should be explained by a substance.

\section{The Unique Properties of the Regular Octahedron Configuration}

When two moving electrons converge, their electrostatic and magnetic forces interact. The electrostatic force produced between the electrons is repulsion; when the direction of motion of two electrons is the same, it brings about an attractive magnetic force. Thus if the direction of motion is the same when two electrons converge, this becomes a special condition, since the stability of the 
system must be maintained.

When the motion track of electrons is projected around the eight vertices on the six surfaces of cube (regular hexahedron), and the direction of motion of electron is drawn (Figure 1), the condition wherein electrons with the same direction of motion converge is satisfied. In the unfurled map, the same number of cube faces denotes synchronization of the track of motion of the electrons projected on these two faces. Because the electron track from an edge comes into a face and comes out from the other edge, the number of entry edges is equal to that of the exit edges, thus the total number of edges of the face must be an even number. All regular polyhedra have only five kinds, with the number of the faces taken separately as $4,6,8,12,20$. This is a mathematical theorem (platonic solids, see Appendix 1). The number of edges of each face in a regular hexahedron is an even number, and other polyhedron is an odd number, thus such structure is unique.

Only the projection of electron motion is reflected above the regular hexahedron. If the circle track of electron motion is to be regarded as a face, then the distances

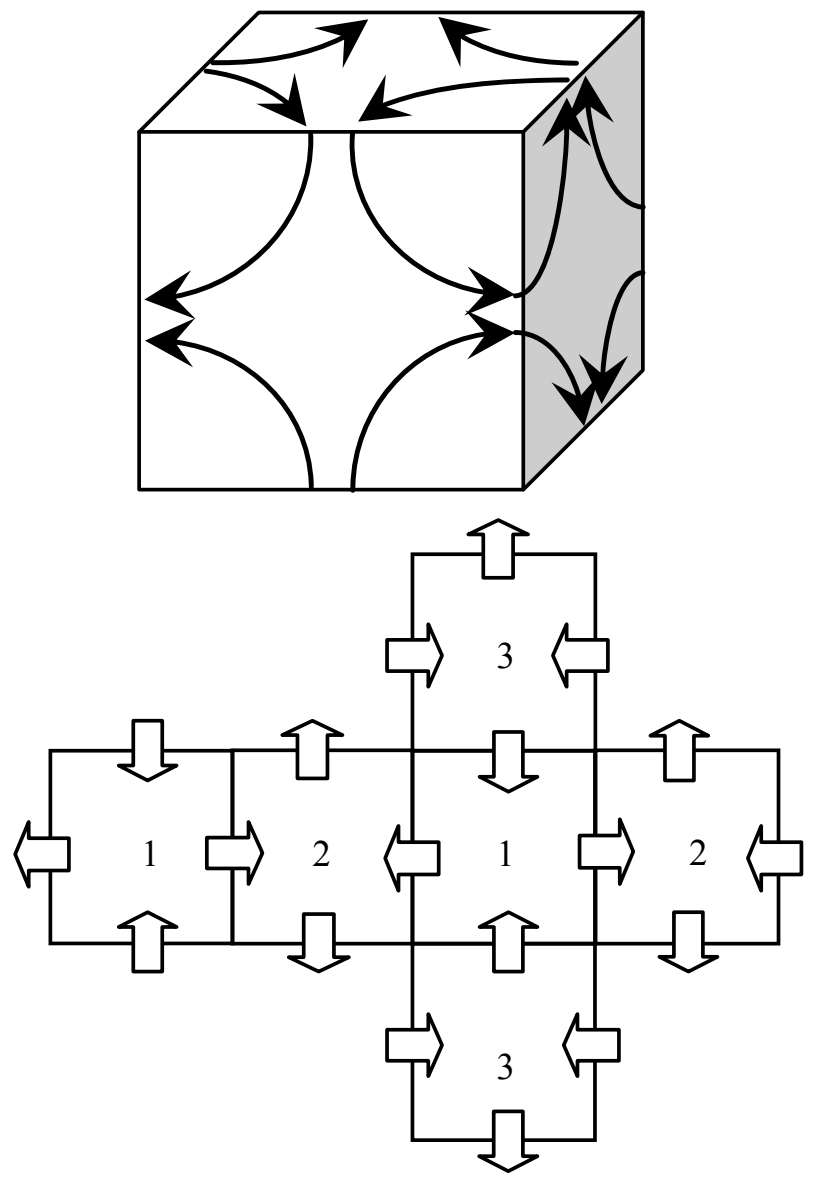

Figure 1. The projection and the unfurled map on the regular hexahedron. from the nucleus to each of the faces are equal. Eight electrons can form a regular octahedron (Figure 2), which only reflects the track of motion of electrons. Electrons converge at the edges of the regular octahedron, satisfying the condition at which electrons converge with the same direction of motion. In the unfurled map, the edges of the same number denote synchronization of the track of motion of electrons at the four edges. Because the electron track from an edge comes into a vertex and comes out from the outer edge, the number of entry edges is equal to that of the exit edges, such that the number of edges of vertex in the regular octahedron must be an even number (see Appendix 1), and other polyhedron is an odd number. This again explains the unique property of the structure. The dual polyhedron of the regular octahedron is a regular hexahedron, which is why this result is obtained.

\section{The Magnetic Moment and Adsorptive Substance of Electron}

Usually, the magnetic force between two electrons is far less than the electrostatic force. Their difference is $v_{\mathrm{e}}^{2} / c^{2}$ factor, where $v_{\mathrm{e}}$ is the speed of electron, and $c$ is the speed of light. Then the magnetic force of electrons that converge on a regular octahedron is too small that it can be ignored, but the fact is not of circumstance. The electron has an intrinsic magnetic moment, if the electron is a small sphere with a crust of electrostatic force, where the charge collects on the surface of the

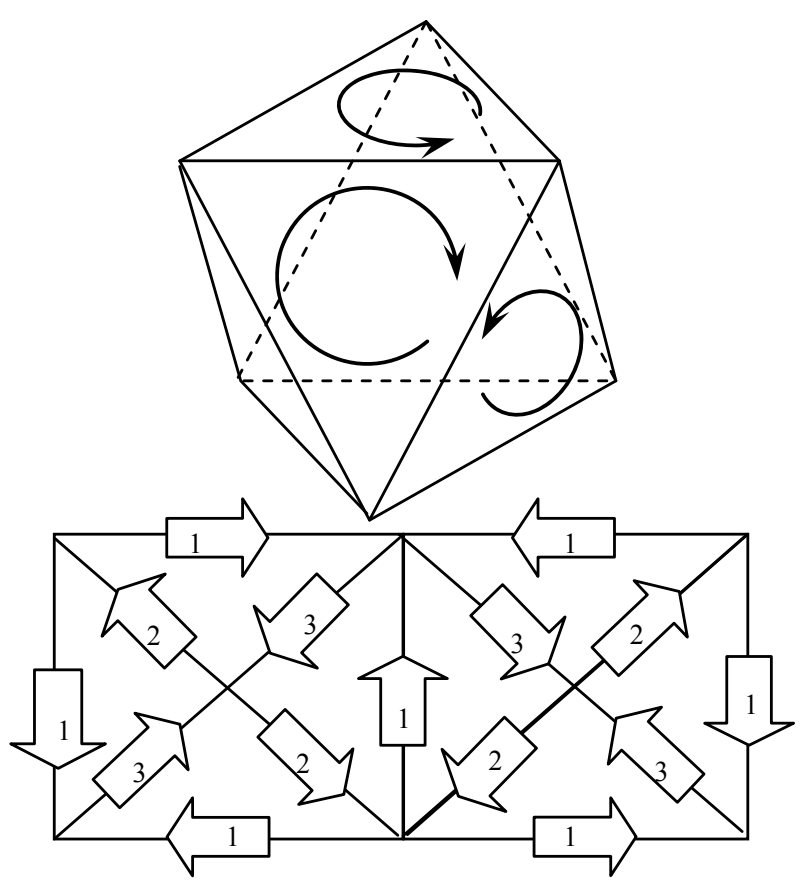

Figure 2. The track on regular octahedron and unfurled map. 
sphere and the spinning produces magnetic moment; then the radius of this electrical property sphere is too small, and the intrinsic magnetic moment is too large: if the value of the intrinsic magnetic moment has to be approximated, the surface speed of sphere will have to greatly exceed the speed of light, many literature referred to this problem. Thus, in terms of distance, the magnetic force and the electrostatic force between two electrons can be compared. Otherwise, if some theory treated the electron as a point particle, this issue may be disregarded, or slide over this question.

Now and here assume that the electron has a magnetic outer layer and an electrostatic force inner layer. The electron exterior is surrounded with a layer of magnetic substance that brings about magnetic force; this layer substance is attracted by the electron that joins and rotates about the electron, producing magnetic force and moment. This is the adsorptive substance of electron: its scope radius is a lot greater than the inner layer radius of the electron. When an electron interacts with another particle outside the scope of the adsorptive substance, it produces electrostatic force, magnetic force, and magnetic moment; if another particle enters into the scope of the adsorptive substance, the adsorptive substance is destroyed or changed, the magnetic force and magnetic moment of the adsorptive substance are eliminated or weakened, and the electrostatic force of electron takes over. This assumption is in contrast with that of exceeding the speed of light with a great magnetic radius of the outer layer of the electron, the magnetic radius $r_{\mathrm{em}}$ of electron being the scope radius of the electron adsorptive substance (Figure 3).

The electron magnetic radius $r_{\mathrm{em}}$ and the magnetic force of the adsorptive substance can be calculated below. The adsorptive substance charge $q$ moves in a circle with velocity $v$ and radius $r_{i}$, the formative magnetic moment $\mu$ is equal to current $i$ multiplied by the circumferential area $S$,

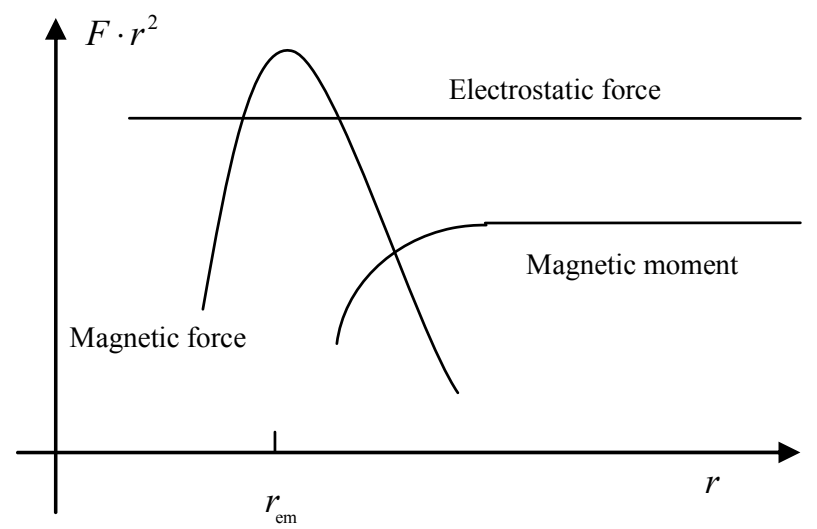

Figure 3. Comparison of electrostatic force, magnetic force and magnetic moment.

$$
\begin{gathered}
\mu=i S=q \frac{v}{2 \pi r_{i}} \cdot \pi r_{i}^{2}=\frac{1}{2} q r_{i} v \\
r_{i}=\frac{2 \mu}{q v} .
\end{gathered}
$$

The adsorptive substance and the electron have equal quantity charge. If the motion of the adsorptive substance is the same as that of electron spin, it will have an electrostatic force; because the adsorptive substance has only magnetic force but not electrostatic force. Thus the adsorptive substance and electron spin axis are in the same plane, the adsorptive substance symmetrically surrounding the electron side and moving in circle with radius $r_{\text {a }}$, and tangential to the inner layer of electron (Figure 4). When the inner layer radius of electron is ignored, $r_{\mathrm{em}} \approx 2 r_{i} \approx 2 r_{\mathrm{a}}$. If not,

$$
r_{\mathrm{em}}=r_{i}+r_{\mathrm{a}}=\frac{2 \mu}{q v}+r_{\mathrm{a}}
$$

Substituted with electron magnetic moment $\mu_{\mathrm{e}}$ and electron charge $\mathrm{e}$ the speed of the adsorptive substance being the speed of light $c, r$ is Compton wavelength $\lambda_{C}$ of electron, the magnetic radius of electron can be computed as follows:

$$
r_{\mathrm{em}}=\frac{2 \mu_{\mathrm{e}}}{e c}+\hbar_{\mathrm{C}}=7.72766 \times 10^{-13} \mathrm{~m}
$$

The distance of the adsorptive substance from the electron spin axis is:

$$
r_{\mathrm{ea}}=r_{i}-r_{\mathrm{a}}=\frac{2 \mu_{\mathrm{e}}}{e c}-\hbar_{\mathrm{C}}=4.475 \times 10^{-16} \mathrm{~m}
$$

Using this method one can also obtain the distance of a proton adsorptive substance from a proton spin axis:

$$
r_{\mathrm{pa}}=\frac{2 \mu_{\mathrm{p}}}{e c}-\hbar_{\mathrm{C}}^{\text {proton }}=3.771 \times 10^{-16} \mathrm{~m}
$$

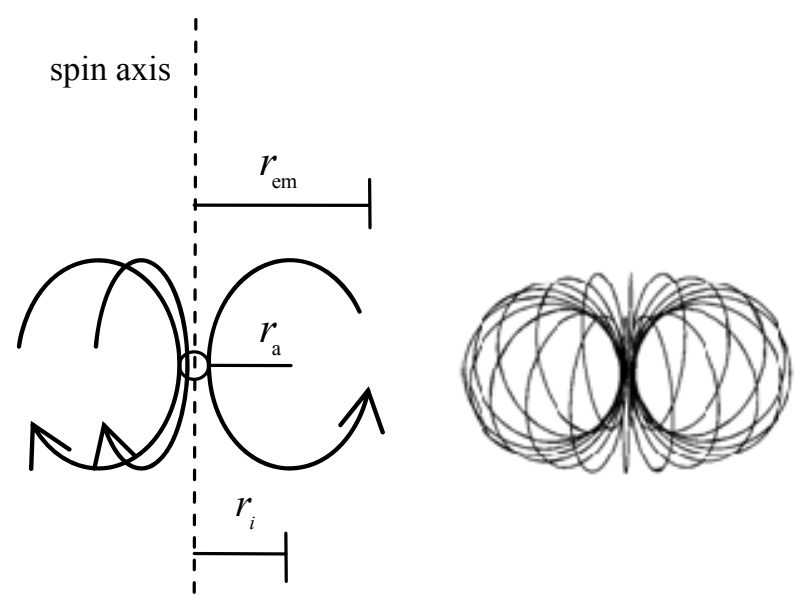

Figure 4. The motion of the electron adsorptive substance. 
where $r_{\text {ea }}$ is on the same order of magnitude as $r_{\mathrm{pa}}$; from here one can also see that $r_{\mathrm{a}}$ is Compton wavelength $\hbar_{\mathrm{C}}$.

Along the circle of the electron spin direction, the ring of the electron adsorptive substance is discontinuous. Suppose $p$ is a positive integer, the adsorptive substance is composed of $p$ circle and the number of petals of electron is $p$.

The electron magnetic moment reveals the quantity of electron adsorptive substance. The atomic nucleus also has an adsorptive substance that can be released, and the electron outside the nucleus absorbs the adsorptive substance that the nucleus released, eventually gaining an angular momentum. The adsorptive substance that is dispersed as a result of collision is converted into radiated heat.

In a hydrogen atom, the proton charge is equal to the electron; the adsorptive substance generated is equal to the quantity of magnetic moment $\mu_{\mathrm{e}}$, but the proton adsorptive substance is released, generating an electron orbit magnetic moment $\mu_{\mathrm{o}}$ and the proton magnetic moment $\mu_{\mathrm{p}}$. The electron with speed $v_{\mathrm{e}}$ is able to release the adsorptive substance, with the generated magnetic moment $\mu_{\mathrm{e}} v_{\mathrm{e}} / c$. A petal is $\mu_{\mathrm{e}} v_{\mathrm{e}} / p c$; its direction is the same as that of the orbit magnetic moment, tangential to the orbit, and participates in generating orbit magnetic moment $\mu_{\mathrm{o}}$, thus

$$
\mu_{\mathrm{e}}=\mu_{\mathrm{o}}+\mu_{\mathrm{p}}-\frac{\mu_{\mathrm{e}} v_{\mathrm{e}}}{p c} .
$$

If $\mu_{\mathrm{o}}$ is Bohr magneton $\mu_{\mathrm{B}}$, then $v_{\mathrm{e}} / c$ is finestructure constant $a$, the number of petals of electron $p$ is 20 , that is, the electron has twenty petals.

$$
\mu_{\mathrm{e}}-\mu_{\mathrm{p}}=\mu_{\mathrm{B}}-\frac{a \mu_{\mathrm{e}}}{20} .
$$

If the electron absorbs more than one adsorptive substance that the nucleus releases each period, then $n$ is a positive integer

$$
n\left(\mu_{\mathrm{e}}-\mu_{\mathrm{p}}\right)=\mu_{\mathrm{o}}-\frac{\mu_{\mathrm{e}} v_{\mathrm{e}}}{20 c} .
$$

The adsorptive substance release and absorption brings about the transfer of the atomic nucleus spin angle momentum to the electron, becoming the electron angle momentum. The electron and atomic nucleus may keep a determinate distance, thus maintaining the stability of the atom.

\section{The Magnetic Force of Electron Adsorptive Substance}

After considering the electron adsorptive substance at a position far from the electron, the magnetic force that the electron motion generates is the same $v_{\mathrm{e}}^{2} / c^{2}$ as the electrostatic force. At a position near the magnetic radius of the electron, the speed of the electron adsorptive substance is equal to the speed of light, with the magnetic force being nearly on the same order of magnitude as the electrostatic force of electron. The adsorptive substance does not have electrostatic force, though it has the same quantity of positive and negative charges and possesses polarity. The polarity rotational frequency and phase of the adsorptive substance tangent to electron, is the same. In a hydrogen atom, after coupling between the magnetic moment of the electron adsorptive substance $\mu_{\mathrm{e}}$ and that of the electron motion magnetic moment $\mu_{\mathrm{B}}$, and with the proton magnetic moment $\mu_{\mathrm{p}}$ with different rotational frequency and phase the relationship is expressed as follows:

$$
\sqrt{2}\left(\mu_{\mathrm{e}}-\mu_{\mathrm{B}}\right)=\mu_{\mathrm{p}} .
$$

If all frequencies and phases are the same, the coefficient will be equal to 2 . When two electrons with the same velocity and direction converge, their adsorptive substances will also have the same rotational frequencies and phases: the magnetic force of motion of electron will correspond to the charge as that of the electrostatic force. Thus, at the magnetic radius of electron, the total magnetic force corresponding to the electron intrinsic magnetic moment is two times the electrostatic force of the electron. At an appropriate direction, the maximum vector sum of the magnetic force is approximately $\sqrt{2}$ times the electrostatic force. This illustrates the case wherein two magnetic moments or magnetic dipole adjoin. When distance $r$ increases, the magnetic force according to $r^{-3}$ likewise decreases. On a regular octahedron, with the edge serving as a boundary that divides the north and south poles, this on a regular octahedron, with the edge serving as a boundary that divides the north and south poles, this arrangement satisfies the two adjoining poles (Figure 5), this requires that the number of edges of each vertical be an even number. Other regular polyhedra cannot satisfy this condition.

The sideways radius of the electron adsorptive substance is $r_{\mathrm{em}}$, and the lengthwise radius is $r_{\mathrm{a}}$. When the distance of two electrons is greater than $2 r_{\mathrm{a}}$, the electrons have the action of both electrostatic force and magnetic moment. When the distance of two electron is near $2 r_{\mathrm{a}}$, the magnetic force may be greater than the electrostatic force. When the distance of two electrons is less than $2 r_{\mathrm{a}}$, the adsorptive substances are changed, and the electrostatic force is greater than the magnetic force. This property of the electron magnetic force provides a basis for the model of the regular octahedron: if two electrons converge with the same velocity and direction, the magnetic force can be equal to or greater than the electrostatic force. 


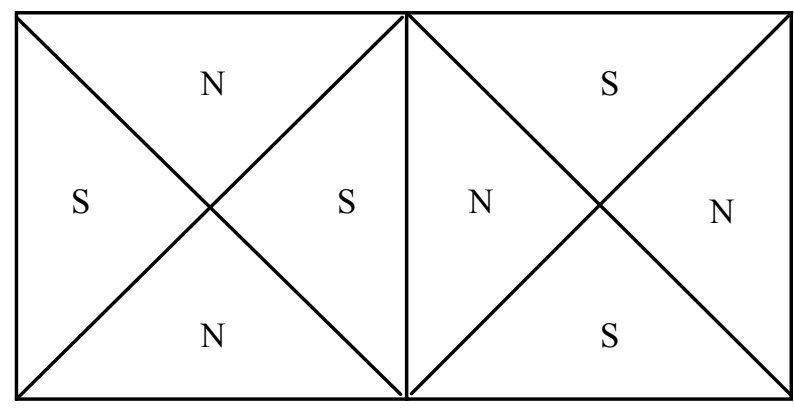

Figure 5. Border upon distribution of north and south poles on regular octahedron.

The magnetic force is two times the electrostatic force, and it also appears in other occasions. The empirical formula of $\beta$ stability line of atomic nucleus is [2]

$$
Z=\frac{A}{1.98+0.0155 A^{2 / 3}} .
$$

Expressing the ratio of the nucleon number $A$ to the proton number $Z$,

$$
\frac{A}{Z}=1.98+0.0155 A^{2 / 3} \approx\left(2-\frac{1}{50}\right)+\frac{A^{2 / 3}}{64} .
$$

The first item explains that at close range, the nuclear force is twice the electrostatic force, the proton number is twice the electron number, $1 / 50$ is caused by the interval of proton and electron. The second item explains that at long distance $r$, the nuclear force according to $r^{-3}$ decreases, the electrostatic force according to $r^{-2}$ decreases; When the nucleon number $A$ is from 50 to 64 , the nuclear force and the electrostatic force at far range are equal, and the atomic nucleus is most stable.

\section{The Regular Polyhedron Configuration}

The regular octahedron configuration is the only regular polyhedron in which the electron motions converge in the same direction. If allow the electrons converge in reverse direction, then all regular polyhedra satisfy this requirement. If converge in reverse direction, electrons move to a common edge at different times, there are not collision. It is reasonable to imagine that the electronic shell of the inert gases is at the second shell to outermost shell, and every shell is composed of a regular polyhedron. In each electronic shell, circles of electronic motion are faces of the regular polyhedron; electronic number is the faces number of the regular polyhedron. Two electrons of the first shell compose two parallel rings, with the electron moving in the same direction in a cylinder (Figure 6). The spins of two electrons are in the same direction, adjoining north and south poles. They can either be the same or in reverse direction of the orbital angular momentum at one time, thus helium has two configurations. The electrons of each shell of the inert gases

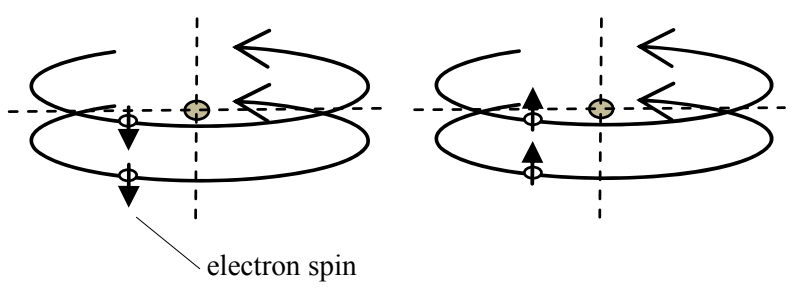

Figure 6. The double ring of same direction and parallel.

element composite configuration and the electron motion modes are as follows:

First shell: cylinder, same direction and parallel;

Second shell: regular octahedron, converge at same direction;

Middle shells: regular polyhedron, face numbers are 6 , 12, 20, allow converge in reverse direction;

Outermost shell: regular octahedron, converge at the same direction.

Thus the electron shell configuration of the inert gases element can be denoted by a row of the face number of regular polyhedron, inasmuch as it is in accordance with the electron configuration of periodic table of elements (Table 1). 18 is dodecahedron and hexahedron, 32 is dodecahedron and icosahedron.

The orbital magnetic moment of the electron also engenders the adsorptive substance. The speed of the same shell electrons is near equation, the electrons can share the adsorptive substance, via its transfer. The energy of each electron tends to equate, and the adsorptive substance even increases the available distance of the magnetic force between electrons. The spin of atomic nucleus extends out and causes the first shell electrons to form a cylinder. For cylinder and regular octahedron, the electron motions of the parallel plane are in the same direction, forming a dipole, consistent with the spin polarity of the atomic nucleus. For hexahedron, dodecahedron and icosahedron, the electron motions of the parallel plane are in the reverse direction, forming a monopole, such as hexahedron with dodecahedron and hexahedron with icosahedron: these combinations can eliminate polarity. The inner shells of electrons are near the atomic nucleus, receive more adsorptive substances that are released by the nucleus, possess a higher negative charge density, and are at the accumulative position of the adsorptive substances. As such, the angular momentum of inner and outer shells is higher, shaping the regular polyhedron with more face numbers. The angular momentum of the middle shells is lower, shaping the regular polyhedron with lesser face numbers.

Before atomic number 21, the regular polyhedron shell and the electron configuration is same in number. The regular polyhedron electronic shells of elements which atomic number are 1 - 103 see Appendix 2. 
Table 1. The regular polyhedra that are composed of inert gases electronic shell.

\begin{tabular}{ccc}
\hline $\begin{array}{c}\text { Inert } \\
\text { element }\end{array}$ & $\begin{array}{c}\text { Electron } \\
\text { configuration }\end{array}$ & Regular polyhedron \\
\hline $\mathrm{He}$ & 2 & 2 \\
$\mathrm{Ne}$ & 2,8 & 2,8 \\
$\mathrm{Ar}$ & $2,8,8$ & $2,8,8$ \\
$\mathrm{Kr}$ & $2,8,18,8$ & $2,8,12,6,8$ \\
$\mathrm{Xe}$ & $2,8,18,18,8$ & $2,8,12,6,12,6,8$ \\
$\mathrm{Rn}$ & $2,8,18,32,18,8$ & $2,8,12,6,12,20,12,6,8$ \\
\hline
\end{tabular}

\section{The Cluster of Inert Gas Elements}

The cluster of inert gas atoms can be described by models represented by regular polyhedrons or cylinders. For the inert gas atoms $\mathrm{Ar}, \mathrm{Kr}, \mathrm{Xe}$, clusters form according to the preferred magic numbers $[3,4]$.

An explanation using a regular octahedron model can be described as follows: as atoms approach, two closer regular octahedrons have an edge superposed; consequently, two atoms share an edge. Each atom (one regular octahedron) has twelve edges, so each atom can join with another twelve atoms. If twelve atoms are directly set on the edges of a regular octahedron, the resulting structure shows defective tightness. The orbital adsorptive substances on the edge are actually shared by two atoms and these adsorptive substances can undergo excursion. To obtain a tight structure, in a regular octahedron, using a plane that passes through an edge and cutting a vertex with an appropriate angle (Figure 7), twelve edges cut twelve times will result in a regular dodecahedron. If the atoms are then set at its pentagon center, in this way, the resulting structure is the tight, and is in agreement with the descriptions in the literature [4].

A Helium cluster [5] is different from other inert gas elements.

The explanation using the cylinder model is described below: because the helium atom is a cylinder, it can arrange along the direction of a circle and the axis, to form different hexagonal structures, even two layer hexagons (Figure 8), the axes of two layers coincide with each other.

\section{The Magnetic Properties of Iron, Cobalt, and Nickel}

Under the frame of quantum mechanics, the origin of metallic ferromagnetism is an issue that is yet to be resolved [6].

The magnetic properties of iron, cobalt, and nickel can be explained through the regular polyhedron electronic shell found in an atom. The regular polyhedron electronic-

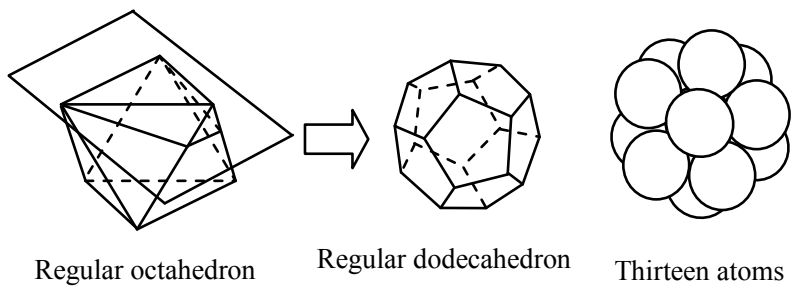

Figure 7. Ar, Kr, Xe cluster.

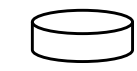

One atom

Cylinder

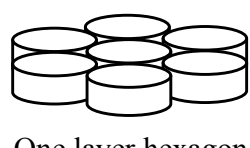

One layer hexagon

Seven atoms

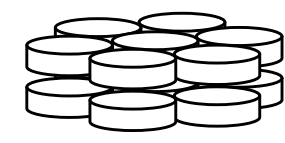

Two layers hexagon
Figure 8. Helium clusters.

shells of iron, cobalt, and nickel are:

$\begin{array}{lll}26 \mathrm{Fe} & 2,8,12,3,1 & 2,8,12,2,2 \\ 27 \mathrm{Co} & 2,8,12,3,2 & \\ 28 \mathrm{Ni} & 2,8,12,5,1 & 2,8,12,4,2\end{array}$

All of these have a regular dodecahedron; this is the origin of the magnetic properties of iron, cobalt and nickel. The respective free or valence electrons of the outermost shells are 1,2,1 or 2, 2, 2; while those of the hypo-outer shell are 3, 3, 5 or 2, 3, 4. The third outer shells are all regular dodecahedrons. Given that hypoouter shell cannot sufficiently form a whole frame. Thus, the hypo-outer shell and third outer shell form a frame of two shells together; the third outer shell of a regular dodecahedron is thereby fixed.

Meanwhile, the motions of electrons on regular dodecahedrons are divided into three cases:

1) On regular dodecahedron, electrons are separated into two hemispheres according to the directions of the electron circumferential motion (Figure 9). At the edges that join the two parts, the directions of the electron motion are the same; there are ten edges which converge with the same direction. Other edges converge from the opposite direction, thereby counteracting the magnetic effect. Ten edges that converge with the same direction engender an equivalent circular electrical current, producing magnetism in the process. Normally, $\mu$ is the magnetic moment of the entire regular dodecahedron. In ten pentagons with a total of ten edges, each edge has two electron paths, and $\mu_{\mathrm{e}}$ is the magnetic moment of each electron, expressed as:

$$
\mu=\frac{1}{5} \times 10 \times 2 \mu_{\mathrm{e}}=4 \mu_{\mathrm{e}} .
$$

2) On the regular dodecahedron of case 1, there is one pentagon each in the center of the two hemispheres; these are marked as A and B in Figure 10. As can be seen, one converges with the same direction, while the other con- 


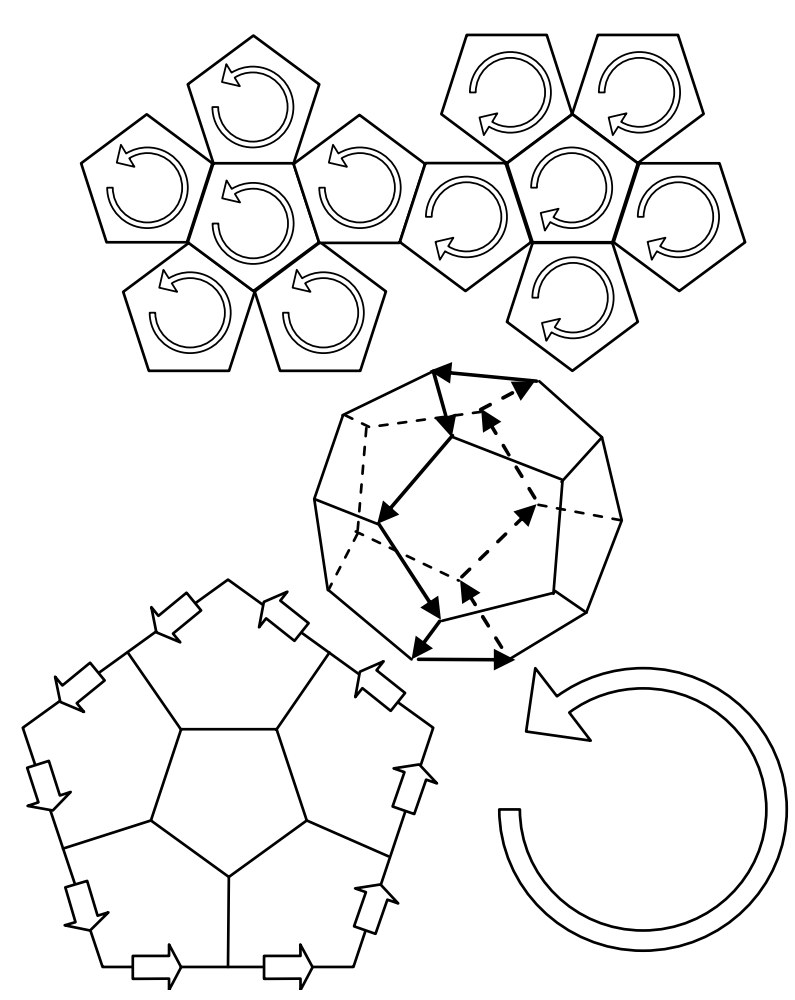

Figure 9. Electron motion and equivalent electrical current in a regular dodecahedron.

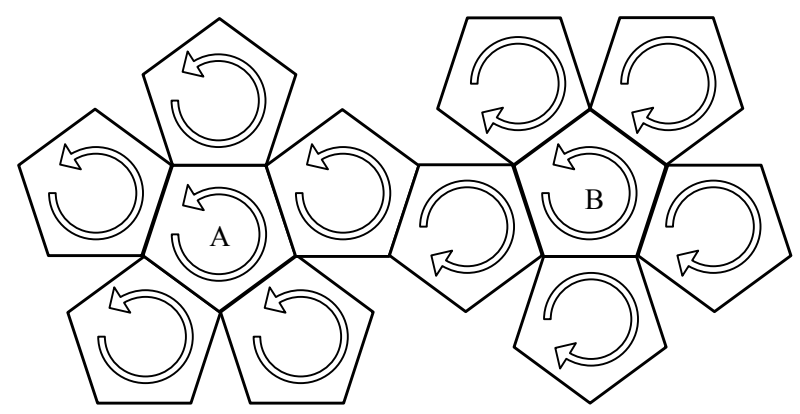

Figure 10. Convergence of the two hemispheres; A, the opposite direction and $B$, the same direction.

verges from the reverse direction:

$$
\mu=2 \mu_{\mathrm{e}} \text {. }
$$

3) Two pentagons in the center of the two hemispheres converge from the same direction as the other edges (Figure 11):

$$
\mu=0 \text {. }
$$

If the efficient Bohr magneton numbers of iron, cobalt, and nickel [7] are expressed by

$n_{\mathrm{B}}^{\mathrm{Fe}}=2.219, n_{\mathrm{B}}^{\mathrm{Co}}=1.715, n_{\mathrm{B}}^{\mathrm{Ni}}=0.604$, respectively, then:

$$
n_{\mathrm{B}}^{\mathrm{Ni}}: n_{\mathrm{B}}^{\mathrm{Co}}: n_{\mathrm{B}}^{\mathrm{Fe}} \approx 1: 3: 4 .
$$

The radii of iron, cobalt, and nickel atoms are very

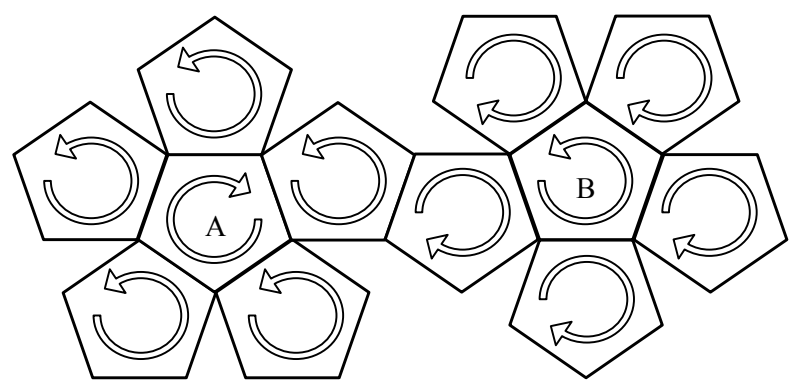

Figure 11. Convergence from the same direction for both A and $B$.

propinquity; thus, the magnetic moment $\mu_{\mathrm{e}}$ of each electron are deemed as equal in a regular dodecahedron.

All atomic structures of iron are case 1:

$$
\mu_{\mathrm{Fe}}=4 \mu_{\mathrm{e}} .
$$

A half atomic structure of cobalt is case 1 , another half atom is case 2 :

$$
\mu_{\mathrm{Co}}=\frac{4 \mu_{\mathrm{e}}+2 \mu_{\mathrm{e}}}{2}=3 \mu_{\mathrm{e}} .
$$

A half atomic structure of nickel is case 2, another half atom is case 3 :

$$
\mu_{\mathrm{Ni}}=\frac{2 \mu_{\mathrm{e}}+0}{2}=\mu_{\mathrm{e}}
$$

From Formulas (17)-(19), we gain:

$$
\mu_{\mathrm{Ni}}: \mu_{\mathrm{Co}}: \mu_{\mathrm{Fe}}=1: 3: 4 \text {. }
$$

Formulas (16) and (20) are concordant.

Therefore the magnetic properties of iron, cobalt, and nickel can be explained by the regular dodecahedron electronic shell of an atom.

The atom radius of iron is $0.126 \times 10^{-9} \mathrm{~m}$, from Formula (2) and $2.219 \mu_{\mathrm{B}}=4 \mu_{\mathrm{e}}$, in the regular dodecahedron shell, the electronic velocity is $c / 289$.

\section{The Thermodynamic Magneton Theory}

The adsorptive substances found in atomic nuclei and electrons are defined as magnetic particles called magnetons.

As a term, "magneton" is commonly used to define or explain a unit of magnetic moment, as in the case of the Bohr and nuclear magnetons, among others. However, in this article, a magneton also denotes a type of particle primarily characterized by a property of magnetism. The magneton is a circle that circumrotates with the speed of light, wherein the radius can change; there are polarities that correspond to the frequency and phase.

The magneton has two different kinds that are separately engendered by either atomic nuclei or electrons; these are called positive and negative magnetons. In an atom, the positive magnetons engendered by the atomic 
nucleus is released and then arranged into a circulative orbit of the electron, thereby forming an orbit positive magneton (Figure 12).

The negative magnetons engendered by an electron have twenty petals and are attracted by electrons. When electrons move, they tend to release negative magnetons. These negative magnetons (1/20) form orbit negative magnetons, while others $(19 / 20)$ turn into ray radiation. Some atoms do not have steady orbit negative magnetons.

In this text, the orbit positive magneton is called an orbit magneton for short. The circulative orbit and velocity of electrons determine the orbit magneton quantity, which is capable of maintaining the redundant orbit magneton's ability to transfer to another atom or transform into heat radiation even when orbit and velocity change. This means that the orbit magneton transforms into heat radiation when it does not have a containable orbit. After some orbit magnetons transform into heat radiation, an increase in electronic momentum will occur. Thus, the electronic orbit becomes capable of holding added magnetons, after which it can stop the process and then transform the magnetons into heat radiation. The frequency of radiation of the negative magnetons is generally higher than the positive magnetons (Table 2).

If the perimeter and density of a magneton are $2 \pi r_{\mathrm{m}}$ and, respectively, then its mass could be computed by $2 \pi r_{\mathrm{m}} D$. The relationship of the polarity periodicity of magneton $N_{\mathrm{mt}}$, the polarity frequency $f_{\mathrm{m}}$, and radius $r_{\mathrm{m}}$ are expressed as:

$$
c f_{\mathrm{m}}=2 \pi r_{\mathrm{m}} / N_{\mathrm{mt}} .
$$

The polarity frequencies of a positive magneton in all static atomic nuclei are equal.

The magneton radius, periodicity, and the positive magnetons released from the proton are also equal. However, in the case of positive magnetons, which are re- leased from the atomic nucleus (with more than one nucleon), the magneton radius and periodicity are not necessarily equal.

Meanwhile, just as there are attractions between the negative and positive magnetons, similarly, there are also attractions between the negative and orbit magnetons. The positive magnetons that are attracted by an electron first accumulate at two ends of the electron spin axis; from there, they form an impetus magneton. The impetus magneton may be integrated stepwise to the inner electrons, released out along the motion of the negative magneton of the electron occurring after break up. It can
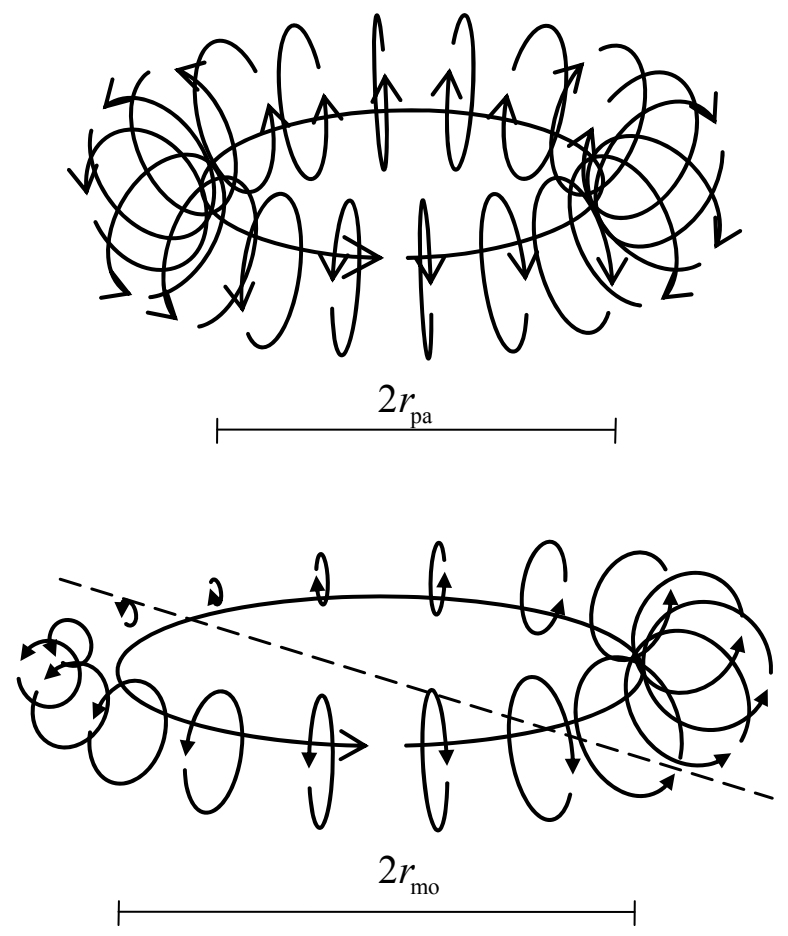

Figure 12. Positive magnetons of proton and orbit positive magneton at electron orbit.

Table 2. Magnetons.

\begin{tabular}{|c|c|c|c|c|}
\hline Magneton & Positive magneton & Negative magneton & Orbit magneton & Negative orbit magneton \\
\hline Polarity & Positive & Negative & Positive & Negative \\
\hline Location & Proton periphery & Electron periphery & $\begin{array}{l}\text { Electron orbit } \\
\text { eriphery }\end{array}$ & $\begin{array}{l}\text { Within electron orbit } \\
\text { circle }\end{array}$ \\
\hline $\begin{array}{l}\text { Engendered by } \\
\text { (transform from) }\end{array}$ & Atomic nuclei & Electrons & Positive magneton & Negative magneton \\
\hline Turn into radiation & Heat low freq. & Ray high freq. & Heat low freq. & Ray high freq. \\
\hline Magneton radius & $r_{\mathrm{pm}}=\lambda_{\mathrm{C}}^{\text {proton }}=2.1031 \times 10^{-16} \mathrm{~m}$ & $r_{\mathrm{em}}=\lambda_{\mathrm{C}}=3.8616 \times 10^{-13} \mathrm{~m}$ & $r_{\mathrm{m}}$ & $r_{\mathrm{m}}$ \\
\hline Magneton orbit radius & $r_{\mathrm{pa}}=3.771 \times 10^{-16} \mathrm{~m}$ & $r_{\text {ea }}=4.475 \times 10^{-16} \mathrm{~m}$ & $r_{\mathrm{mo}}$ & $r_{\text {mo }}$ \\
\hline
\end{tabular}


then cause the electron to obtain impetus. As many positive magnetons accumulate, these compound into a circlet which becomes the compound positive magneton of the large circle. Here, the polarity periodicity of the compound is the average value before the magnetons compound. If the radius of the compounded positive magneton is greater than the diameter of the negative magneton of an electron, then the compound positive magneton shall leave two ends of the electron spin axis and transfer in a parallel position to the side of the electron's negative magneton. This combination of compound positive magneton becomes the orbit magneton. The rules of interaction among positive, compound, and orbit magnetons are explained below (Figure 13):

1) If two orbit magnetons have equal polarity periodicities and radii, the spin axes are in superposition and the spin direction are the same, then there would be no force action; if the spin axes are in superposition but the spin direction is in reverse, then there would be an at traction. On the other hand, if the circles of two magnetons border upon the same plane and the spin direction is the same, then there would be an attraction; in this case, the linear velocity at adjacent point is in reverse direction.

2) If two orbit magnetons have equal polarity periodicities but unequal radii, their spin axes are in superposition, and if the spin direction is the same, then there would be an attraction; but if the spin direction is in reverse, then there would be a repulsion. If the circles of two magnetons border upon the same plane and the spin direction is in reverse, then there would be an attraction; in this case, the linear velocity at adjacent point is the same direction.

3) If two orbit magnetons have unequal polarity peri-
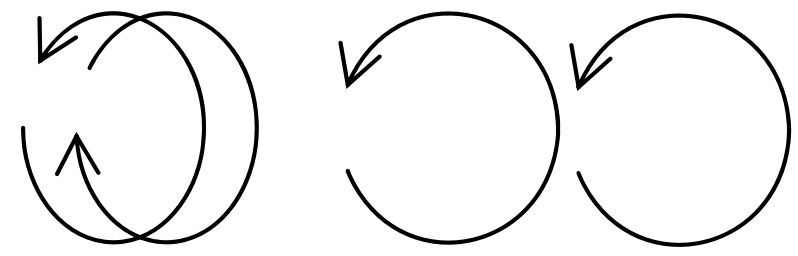

Rule 1

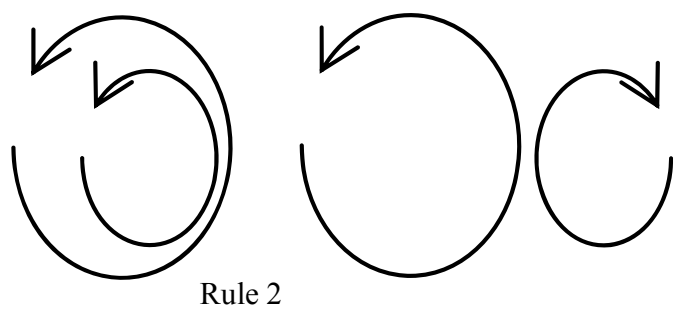

Figure 13. Attraction of orbit magnetons. odicities, there would be no force action.

If a multi-orbit magneton forms a ring, the circle of each magneton would be perpendicular to the electron orbit (Figure 12). The orbit direction with similar spin directions and arrays would form into a single team or one with a reverse spin direction according to the radii (from large to small). If two magnetons form at the junction and the radii are equal, then the largest or smallest in the team will separate. If the orbit magneton ring on the electron orbit forms 2, 4, 6 and even more teams (two teams at the very least) and the magneton spin directions of the two adjacent teams are in reverse, this will lead to a magnetic moment of zero at the exterior. In Figure 12, the broken line separated two teams.

For the electronic shell of regular polyhedron, the orbit magneton distribute in the edge of regular polyhedron.

If the electronic motion does not periodically turn around, then the orbit magnetons would no longer form a ring so that the beginning would connect to the end. Beside the electron, two teams of orbit magneton would form an olive shape (Figure 14).

When many atoms or molecules come in contact with each other, some parts of the action between electrons are processed via the orbit magnetons. If the orbit magnetons maintain stable attraction, then the object would be in a solid state. The attraction of the orbit magnetons can decrease the distance between electrons as the electron's negative magneton show no apparent attraction. If the distance between electrons is shortened, this would cause a fraction of the orbit magnetons to be pushed out, leading to slippage between atoms or molecules; if this is the case, then the object is said to be in a liquid state. If most of the orbit magnetons are pushed out and the atoms or molecules are also bounced out, then the object is said to be in a gaseous state.

The attraction between the orbit magnetons is in direct ratio with the amount of magnetons. This is also related to the polarity periodicities and radii of such magnetons.

In the gaseous state, the orbit magnetons that are pushed out when molecules come in contact with each other and bounce out become free positive magnetons. The bounced out molecules can also capture free positive

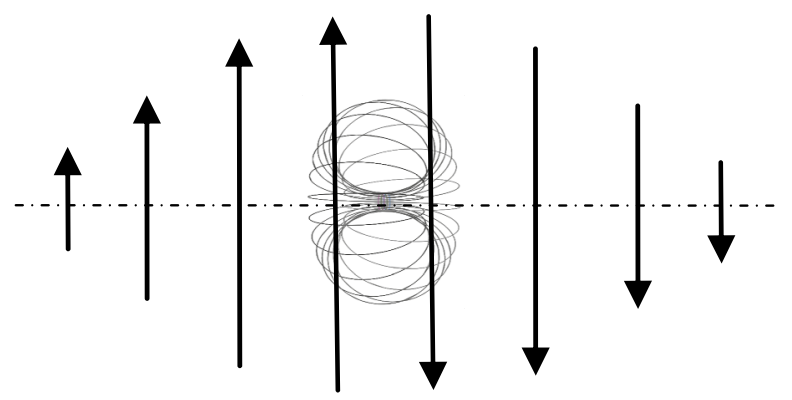

Figure 14. The olive-shaped orbit magneton. 
magnetons and may become orbit magnetons. The overall velocity of the free positive magnetons is equal to zero. After the molecule captures the positive magnetons, the speed of the molecule would then be reduced. If molecules have same orbit magnetons, then their speeds are the same.

The action of molecules in the positive magnetons is separated into the force of the free positive magnetons $f$ and the force of the captured orbit magnetons $F$.

If the attraction of molecules in the free positive magnetons is $f$, the mass of the free positive magnetons is $M$, and the acceleration is $a$, then we have:

$$
a=\frac{f}{M}
$$

If the attraction of molecules in the captured orbit magnetons is $F$; when captured, the attraction is $\mathrm{d} F$, the mass is $\mathrm{d} M$, and the acceleration is the same as the acceleration of the free positive magnetons $a$, then:

$$
\mathrm{d} F=a \mathrm{~d} M=\frac{f}{M} \mathrm{~d} M .
$$

In an equilibrium state, the $f$ of every molecule would be equal. If the molecules with equal $\mathrm{d} M$ are considered as the same kind, then $F(M)$ is the distribution of the attraction according to the same kind of molecular total in $M$ stated as:

$$
F(M)=\int a \mathrm{~d} M=\int \frac{f}{M} \mathrm{~d} M=f \ln M .
$$

On the basis of the inter-actional rules of orbit magnetons, only the same kind of molecules may attract each other and push out the orbit magneton; in this situation, the system tends to increase as many different kinds of molecules as possible and capture the maximum positive magnetons.

When molecules bounce out, the work of repulsive force equals the negative work of attraction, expressed by the attraction $F$ multiplied by the maximal radius of orbit magneton $r_{\max }$. Meanwhile, the kinetic energy when molecules bounce out is equal to the work of the repulsive force,

$$
E_{\mathrm{k}}=-F r_{\max } \text {. }
$$

From Formulas (24) and (25), we derive:

$$
\begin{aligned}
& E_{\mathrm{k}}=-f r_{\max } \ln M, \text { and } \\
& M=e^{-E_{\mathrm{k}} / f r_{\max }} .
\end{aligned}
$$

This is the relation of the free positive magnetons mass $M$ and the kinetic energy of molecular $E_{\mathrm{k}}$. As compared with the Boltzmann factor $e^{-E_{\mathrm{k}} / k T}$, we can see that the Boltzmann constant $k$ is the attraction of molecules for the free positive magnetons $f$. If it is constant in the equilibrium state, the temperature $T$ is the maximal radius of orbit magneton $r_{\max }$, expressed as:

$$
\begin{gathered}
k=f, \text { and } \\
T=r_{\text {max }} .
\end{gathered}
$$

The dimension of $k T$ is work, the dimension of temperature $T$ is length, and the dimension of $k$ is force. From this relationship, we can say that the magneton theory can work well with thermodynamics.

The maximal radius of the impetus magneton is equal to the minimal radius of the orbit magneton $r_{\min }$, and is also equal to the radius of the negative magneton on static electron $r_{\mathrm{em}}$. The temperature $T$ defined by perfect gas is a macroscopic and statistical quantity of multiunits (molecule); meanwhile, the temperature $T$ of an area near zero $\mathrm{K}$, that is equivalent to: in this area, there are almost no orbit magnetons of the outer shell that may lead to a shift out. Therefore when temperature $T$ nears zero $\mathrm{K}$, the outer shell of most units will have not orbit magnetons; for the few units that have orbit magnetons in the outer shell, the radius of the orbit magneton will take a minimum value as expressed by:

$$
T=r_{\max }=r_{\min }=r_{\mathrm{em}}=\lambda_{\mathrm{C}} \text {. }
$$

In the outer shell of metal, electrons are far apart from the atomic nucleus, and the number of absorbed positive magnetons could be low such that these would not form into a fixed frame. In such a case, it can rely on the secondary outer shell electrons so that it could be joined together; this is called the hypo-outershell. The outer shell electrons become free electrons in the frame interstice (a free electron has a small quantity of impetus magneton and has an initial kinetic energy $\left.E_{0}\right)$. The action between the free electrons is similar to what is occurring in the gaseous state; also, if the free electron has the action of another item from the hypo-outer shell frame via free positive magneton, Formulas (22) and (25) can be changed to:

$$
\begin{gathered}
a=\frac{f}{2 M} . \\
E_{\mathrm{k}}-E_{0}=-2 F r_{\max } .
\end{gathered}
$$

Using a coefficient $i$ denotinge the scale of two item's action, $0 \leq i \leq 2$, and from Formulas (24) and (32), we derive:

$$
\begin{aligned}
& E_{\mathrm{k}}-E_{0}=-i f r_{\max } \ln M, \text { and } \\
& M=e^{-\left(E_{\mathrm{k}}-E_{0}\right) / i f r_{\max }} .
\end{aligned}
$$

Suppose $m_{\mathrm{e}}$ is the electron mass, $v_{\mathrm{e}}$ is the electron velocity, $v_{0}$ is the initial velocity of electron, the momentum of the action between the electron and hypoouter shell frame is $m_{\mathrm{e}} v_{\mathrm{e}}$, and $t$ is the acting time:

$$
m_{\mathrm{e}} v_{\mathrm{e}}=F t=-f t \ln M \text {, and }
$$




$$
M=\mathrm{e}^{-m_{\mathrm{e}} v_{\mathrm{e}} / f t} .
$$

From Formulas (34) and (36), we derive:

$$
\begin{gathered}
-\left(E_{\mathrm{k}}-E_{0}\right) / i f r_{\max }=-m_{\mathrm{e}} v_{\mathrm{e}} / f t, \\
\frac{\left(\frac{1}{2} m_{\mathrm{e}} v_{\mathrm{e}}^{2}-\frac{1}{2} m_{\mathrm{e}} v_{0}^{2}\right)}{i f r_{\max }}=\frac{m_{\mathrm{e}} v_{\mathrm{e}}}{f t}, \text { and } \\
t=2 i r_{\text {max }} \frac{v_{\mathrm{e}}^{2}-v_{0}^{2}}{v_{\mathrm{e}}} .
\end{gathered}
$$

The electrical resistance of metal mostly comes from the action occurring between the electrons and the hypoouter shell frame. It is derived via the transfer of free positive magnetons, electrical resistivity $\rho$ is namely the acting time $t$, and the maximal radius of the orbit magneton $r_{\max }$ is the temperature $T$, which is treated $i$ as the probability of all collision. Hence, the collision probability between electrons is $P_{\mathrm{c}}=i / 2$,

$$
\rho=4 P_{\mathrm{c}} T \frac{v_{\mathrm{e}}^{2}-v_{0}^{2}}{v_{\mathrm{e}}} .
$$

If $v_{\mathrm{e}}<v_{0}$, or $P_{\mathrm{c}}=0$, then it is said to be in a superconductive state. The directional motion of the electron can be maintained in the initial kinetic energy $E_{0} . P_{\mathrm{c}}=0$ is the non-collision superconductivity, and $v_{\mathrm{e}}<v_{0}$ the superconductivity when collision speed is less than the initial velocity.

When the temperature $T$ is lower, consider two special cases below:

1) If $P_{\mathrm{c}}$ and $v_{0}$ are close to the constants, then

$$
\rho \sim T\left(v_{\mathrm{e}}-\frac{A_{1}}{v_{e}}\right) .
$$

As such, $A_{1}$ is approximately constant. From Formula (33) we derive $T \sim v_{\mathrm{e}}^{2}$, so

$$
\rho \sim T\left(\sqrt{T}-\frac{A_{1}}{\sqrt{T}}\right) .
$$

2) If $v_{\mathrm{e}}$ and $v_{0}$ are close to the constants, the mass of the olive-shaped orbit magnetons would be proportional to the square of the magneton radial; thus, theattraction between electrons would be proportional with the collision probability, $P_{\mathrm{c}} \sim T^{2}$, leading us to the following:

$$
\rho \sim T^{3}
$$

When the temperature $T$ is higher, then $v_{\mathrm{e}} \gg v_{0}$. According to the magneton interactional rule, the free electrons attract each other but only if they have the same speed. If there is an increase in free electrons with different speed levels, the range of speed would increase and the collision probability will decrease, $\rho \sim\left(1 / v_{\mathrm{e}}\right)$, so

$$
\rho \sim T
$$

Therefore, the part of electrical resistivity $\rho>0$ can disport three segments; these are shown in Formulas (42) to (44). For the expression $\rho \sim T^{3}$ see also [8].

The high critical temperature superconductivity can be seen in non-collision superconductivity. To increase the critical temperature, the following methods are followed:

1) Release the free electrons from the atoms of multiple sorts.

2) The atomic nucleus can release the positive magnetons with different periodicity, these positive magnetons can then form the orbit magnetons of multiple sorts.

3 ) Increase the speed range of the free electrons.

4) Restrict the motion range of the free electrons.

5) Decrease the density of the free electrons.

In Method 2, the first requirement is an element with a high melting point and the second is an element with low rigidity.

In Formulas (26) to (29), the Boltzmann factor $e^{-E_{\mathrm{k}} / k T}$ is equal to the free positive magnetons mass $M$. In some liquids, $M^{-1}$ can be viewed as a macroscopic amount referring to viscosity $\eta$. In $[9,10]$,

$$
\eta=A e^{Q / R T} .
$$

where $Q$ is the activation energy, $R$ is the molar gas constant, and $A$ is a constant. If one molecule is transformed to many molecules, then $k$ is transformed to $R$, and $E_{\mathrm{k}}$ is transformed to $Q$. In these liquids, the viscosity $\eta$ is inversely proportional to the free positive magnetons mass $M$ in the area:

$$
\eta \sim M^{-1}
$$

In $[11-13], \eta=A e^{B / R T}\left(1+C e^{D / R T}\right)$, where $A, B, C$ and $D$ are constants, then this is a case when there are two types of free positive magnetons. Fermi distribution is a case when there are two types of attractive particle, Bose distribution is a case when there are two types of repulsive particle.

There is a decrease in free positive magnetons in the process of transformation from liquid to solid state; Its part transform into the orbit magnetons $M_{\mathrm{o}}$; if their total $M_{\mathrm{t}}=M+M_{\mathrm{o}}$, then $E$ is a constant:

$$
\eta=E\left(M_{\mathrm{t}}-M_{\mathrm{o}}\right)^{-1} \text {. }
$$

In the presence of orbit magnetons, similar particles can attract each other and molecules and electrons also behave in this manner. In such a case, the ion is also similar. When the glass of oxide $\left(\right.$ as $\left.\mathrm{SiO}_{2}\right)$ change from a liquid to solid state, though free positive magnetons decreasing, there are yet enough orbit magneton, the same ions will attract each other and can separately form an ion group of a similar type. The oxygen ions can then attract each other and form a group of oxygen ions; the 
silicon ions can attract each other and form a group of silicon ions. There are electrostatic attractions between the groups of oxygen and silicon ions. The structure of a glass is formed when a large amount and space of groups of oxygen ions connect and form a net where a group of silicon ions are embedded.

The chemical reaction is regarded as that occurs the electronic transfer, because only the electron those sort and velocity are same attract each other, the course of electron transfer from a sort of atom to another sort of atom, except electrostatic force require also satisfy two condition: first the electronic velocity is same, second is the mix of magneton: the magneton on electron come from several atomic positive magneton, form the orbit positive magneton on electron after mixed. The magneton mix also may be the change of magneton supply, the source of positive magneton from a sort of atom change to another atom. The magneton mix also may the electronic velocity go to equal. Usually the magneton mix easy in high temperature and pressure, and the electronic kinetic energy easy over the electrostatic force, the chemical reaction easy progress. In the situation of temperature and pressure are not change, the magneton mix becomes mostly factor that influence the chemical reaction.

\section{Magneton and Electromagnetic Induction}

The magneton of electron formerly was circle. When two electrons approaching to a scope of distance, the magneton interact and being distorted, the circle plane occur bend; if the distance of two electrons are not changed anew, the bend of circle keep and are not change; at the farther position there is away the central electron, on the edge, the plane of magneton rotated a angle, the angle between circles become uneven. Formerly the magneton moving along circle with the velocity of light, this the tangent direction, the magneton is tangent with the central electron also, both spins keep be in mesh in certain phase; the circle of magneton swing in other direction when distortion, the advance speed is not speed of light in tangent direction, but is less than speed of light, the electron must moving backward and keep be in mesh. This backward motion of electron is the electromagnetic induction (Figure 15).

The motion and spin axis direction of electron incline to superposition when the electromagnetic induction. Two electrons appear the electromagnetic induction; firstly the magnetons of electrons attract each other, the spin axis of electrons incline to same direction, and then the magneton swing, the electron moving backward. Two conditions which the free electron can electromagnetic induction is also two sort swings: the swing of the spin axis of electron and the swing of magneton. The swing of the spin axis cause the spin axis of electrons turning to same direction; the swing of magneton cause the electron that keep be in mesh with magneton moving backward. The electromagnetic induction is the change of the magnetic force causing the swing of magneton of electron, the electron moving, this induction differs from the magnetic force (Figure 16).

Two relation of direction are reverse: the relation of the spin axis of electron with the act direction when electromagnetic induction; and the relation of the spin axis with motion direction when thermal motion.

A motion electron comes into magnetic area; firstly the spin axis is turned, the turned place always got behind with interact point, the spin axis incline to same direction with the electron of the back magnet, and then the magneton swing, the electron plus a motion that moving backward along the spin axis, and is the reverse of electron of the back magnet, it is close perpendicular to the motion direction, but is not completely perpendicular to the motion direction. A motion electron is affected by magnet, the force that effecting electron $f_{\mathrm{m}}$ with

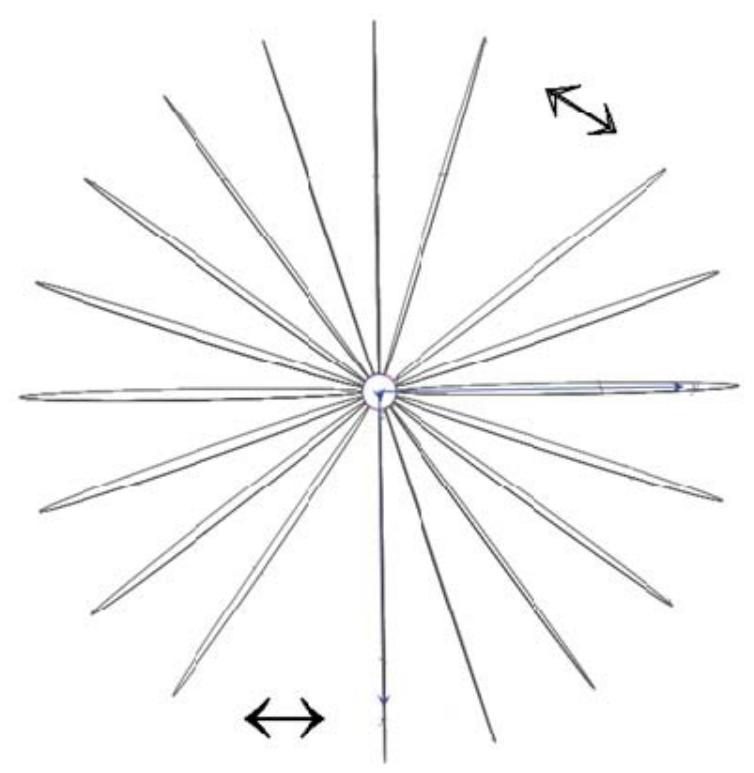

Figure 15. The swing of magneton.

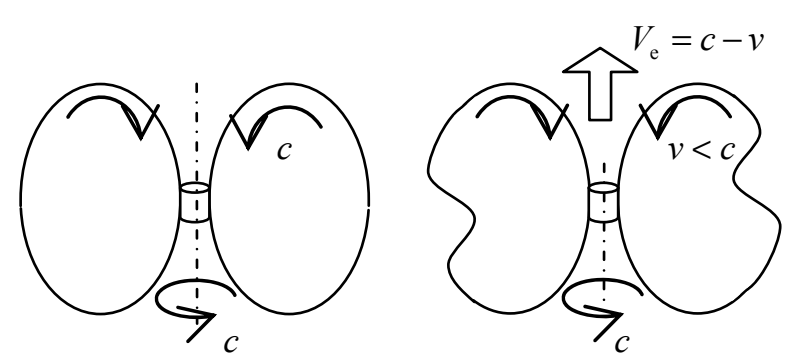

Figure 16. The swing of magnetons causes the electron moving. 
the motion direction of electron (or velocity $v_{\mathrm{e}}$ ) forms an obtuse angle $\partial>90^{\circ}$ (Figure 17).

The magnetic induction satisfies three conditions:

1) The spin axis of electron turning.

2) A revolving ring with velocity of light exists in electron.

3) The swing of revolving ring.

The momentums of instantaneous Lorentz force are not conservation, its action force and effect is perpendicular; the electromagnetism induction satisfies three conditions above are momentums conservation.

The delay and inertia of the electromagnetic induction are greater than the energy of ionization, this explain also three conditions above.

The electromagnetic induction can engender voltage, and engender current if there is a loop. When the area of an electric lead circle changed, each part that lead moving engenders voltage, the directions of these voltages are same; the perimeter of circle changed simultaneity, the perimeter has difference, the voltage to the perimeter difference are in direct ratio, the current is that the perimeter difference multiplies the amount of electron, the perimeter difference to the amount of electron are in direct ratio, the area difference to the square of the perimeter difference are in direct ratio, therefore the current to the area difference are in direct ratio.

\section{The Electronic Shell of Carbon}

The electronic shell of the carbolic atom is 2, 4. Four electrons of the outermost shell formed a regular tetrahedron.

In the regular tetrahedron of atom of diamond, all four electrons converge from reverse direction; each atom is connected by superposition of vertex, that is share four vertexes, therefore each atom connect with other four atoms, and is symmetrical (Figure 18).

In the regular tetrahedron of atom of graphite, three electrons is converged from reverse direction, these three electrons is converged from same direction with the other one electron, in six edges of the regular tetrahedron, there is three edges of one face is converged from same direction, other three edges is converged from reverse direction. Each atom is connected by superposition of three

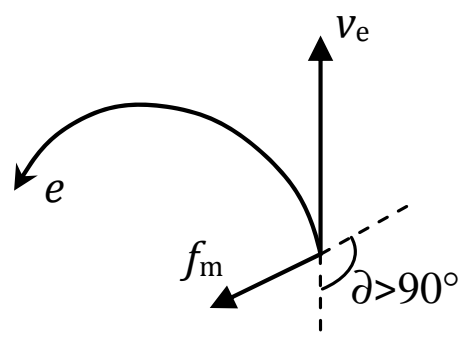

Figure 17. A motion electron is affected by magnet. edges, which are converged from same direction, so each atom is connected with other three atoms, and is in a same plane (Figure 19), these atoms can connect into hexagon.

The converged electron orbit has two sorts of the same or reverse direction from the rule of magneton attract each other: when converge from same direction, the magneton of same radius attract each other; when converge from reverse direction, the magneton of different radius attract each other. The negative magnetons attract each other when converge from same direction, cause the electron orbit close up, and push out the orbit magneton (Figure 20); the negative magnetons do not have attract when converge from reverse direction, electron orbit the do not near mostly, and do not push out the orbit magneton (Figure 21). The electron of atom of diamond all are converge from reverse direction, electrons of adjacent atom are also converge from reverse direction, therefore do not push out the orbit magneton, have some attraction, and equipoise with electrostatic force of nucleus, the electron orbit is most stable.

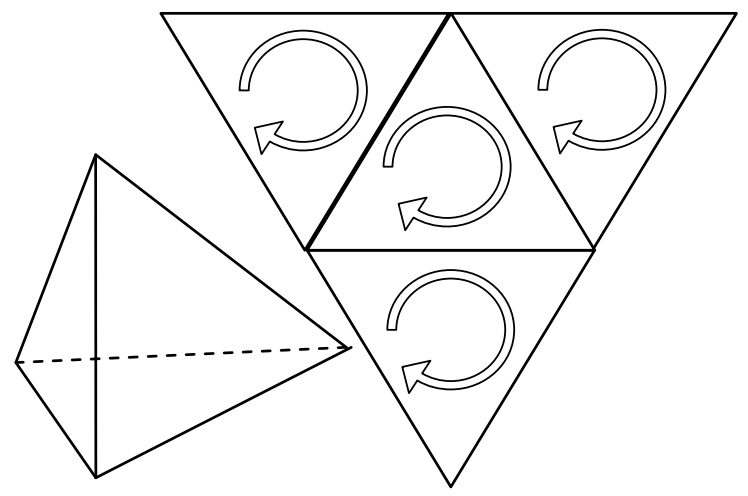

Figure 18. Diamond: four electrons converge from reverse direction.

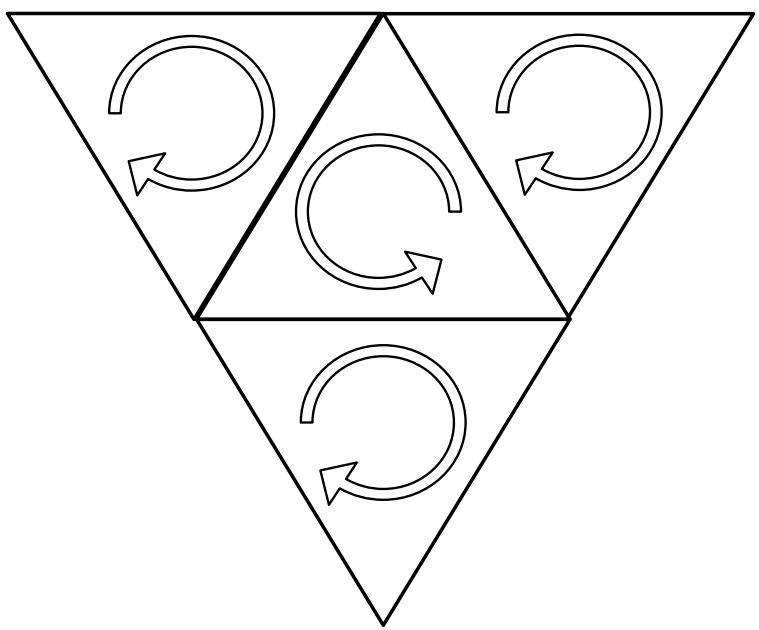

Figure 19. Graphite: three edges of same plane converge from same direction. 

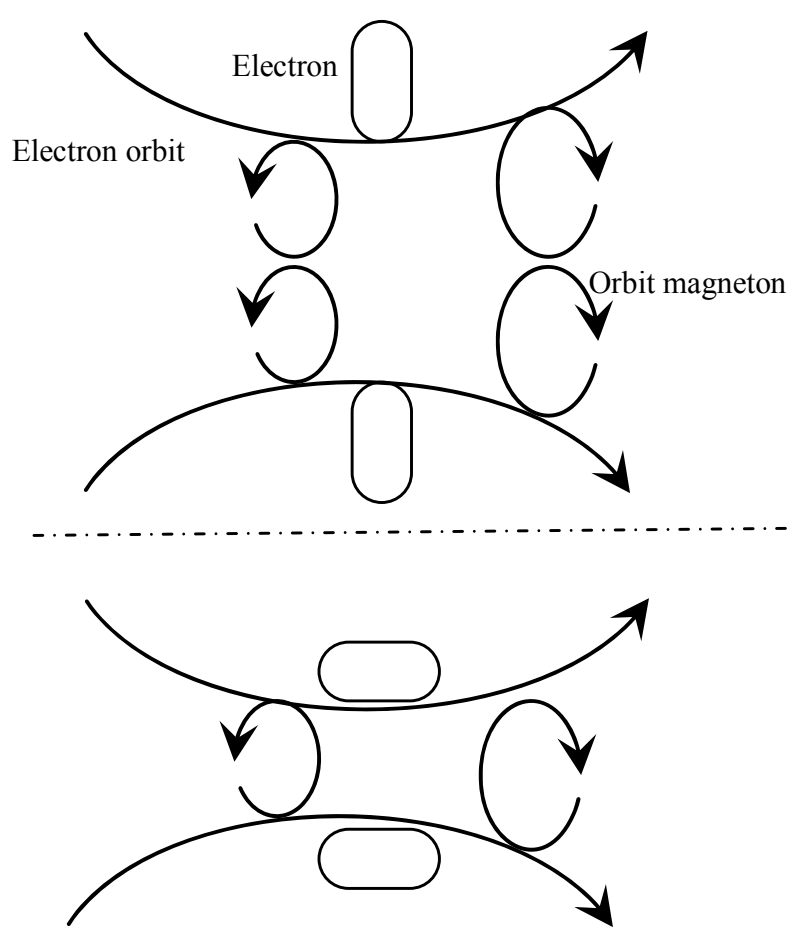

Figure 20. Converge from same direction.

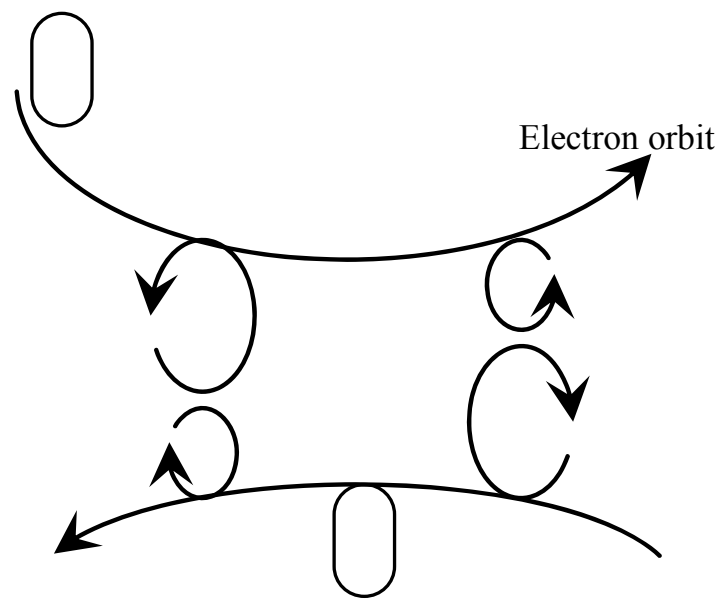

Figure 21. Converge from reverse direction.

\section{The Helical Line Model of the Light}

The structure of the light is a helical line. The axis of the light helix is same from the direction of motion of light. The direction of motion of light is its direction of travel or propagation direction. $\beta$ is the helix angle of the light helix, $45^{\circ} \leq \beta \leq 90^{\circ}$. The helix angle approach $45^{\circ}$ if the light density is high; when the light density debases, the helix angle increase, maximum approach $90^{\circ}$, the helix almost become a straight line. The wavelength of light is the screw pitch of the helix. If the helix angle of the light helix is already $45^{\circ}$ and the light density continue to increase, then the screw pitch of the helix change into short, that is the wavelength of light change into short (Figure 22).

Two planes that are parallel with axis cut symmetrically helix, split helix into three parts, the middle part is a polarized light; if remove the middle part, two remnant parts are also a polarized light.

The light in a magnetic field, the advance have a resistance; the subsequent part of the light helix has an act of pushing for the front part. In the act of resistance and pushing, the front part of the light helix comes into deflection; the deflection of the front part leads the subsequent part and comes into deflection. Therefore the light have a deflection in a magnetic field, the deflection of polarized light can easy be observed.

When a light come close and into an object, have an act first with the magneton of object.

Compton Effect is the result of act of the light and the negative magneton. When light get across the negative magneton, if the axis of the light helix and the spin axis of the negative magneton is superposition, then the helix has a repulsive force, the helix is expanded, the radii of the helix adds the radii of the negativemagneton, that is Compton wavelength $\lambda_{C}$ of electron; the screw pitch of the helix adds the perimeter of the negative magneton $\lambda_{C}$, so the wavelength of light adds $\lambda_{C}$.

The orbit positive magneton included two teams of positive magneton with reverse spin, when the light meets the orbit positive magneton, if the axis of the light helix is perpendicular to the circle of the positive magneton, then the light is reflected; if the axis of the light helix is parallel to the circle of the positive magneton, then the light gets across the positive magneton, come into being refraction.

When the velocity of electron change, the radii of the negative magneton of electron decrease, the superfluous substance of the negative magneton become mostly the radiation of light, the few become the orbit negative magneton of electron. Along the straight line if count the frequency of light; along a circle if count the frequency of magneton. A light can have an act with the orbit negative magneton that the frequency is same, and then is absorbed by the atomic nucleus, translate into the positive magneton. The translation about the magneton and the radiation of light or heat express below:

The positive magneton on the atomic nucleus $\rightarrow$ The orbit (positive) magnetons on the electron orbit $\rightarrow$ The radiation of heat.

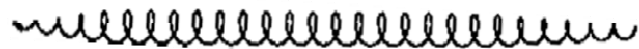

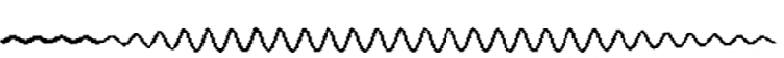

Figure 22. The helical line structure of the light. 
The positive magneton on the atomic nucleus $\rightarrow$ The negative magneton on the electron.

The part of negative magneton on the electron $\rightarrow$ The radiation of light, the orbit negative magneton.

The radiation of heat, the radiation of light + the orbit negative magneton with same frequency $\rightarrow$ The positive magneton on the atomic nucleus.

When the magneton that mass is $m$ transform into the radiation of light of half periodic, the velocity of magneton become the velocity of light $c$, the released kinetic energy is $\frac{1}{2} m c^{2}$.

The light can separate and form again. The diffraction is that the part of light helix is obstructed. The diameter of the light helix is less a little than aperture, the light passing through immediacy if it is at the center of the aperture; the part is obstructed if the light helix is not at the center of the aperture, and change in the directions. The diffraction of twain gap is that the separate light forms again if these phase is same.

The light form again inside the object can explain the color combination. If the diameter of a light helix is less a several fold than another light, a light helix enter into the another light helix inside, the frequency of the combination light is add up, this is the combination of attraction; if the diameter of a light helix is less a little than another light, the frequency of the combination light is reduced, this is the combination of repulsion.

\section{The Maximal Shell Numbers of the Electronic Shells}

The negative magnetons on the electron have twenty; the positive magnetons on the atomic nucleus have twenty also, these positive magnetons release out and become the orbit magnetons in the electron orbit, the positive and negative orbit magnetons change to positive magnetons on the nucleus via action; in this cycle, the atomic nucleus release twenty positive magnetons each period. Because the static attraction, the electron will fall on the nucleus if have not the supplement of the positive magnetons, the nucleus change to other nucleus by the action of the electron and nucleus. The number of the positive magnetons that the nucleus released has decided the maximal shell number of the electronic shells, twenty positive magnetons can support the maximal shell number is ten layer, the electronic shells if the shell number exceed ten layer, the electron fall easily on the nucleus, the nucleus is very unstable. If the atomic number is greater than 90, the electronic shells begin exceed ten layer, the most outer shell electrons already cannot form a whole frame, the electrons have no longer subdivide layer. If the atomic number is more, the electron fall easily on the nucleus and bring the nucleus change.

\section{Conclusion}

Inert gas atoms form a cylinder and a regular polyhedron, forming the same shell electrons. The circle tracks of electrons motion are faces of polyhedron. The electronic number is the faces number of the regular polyhedron. The periodic table of elements is arranged based on a series of regular polyhedrons. The electron has a magnetic outer layer and an electrostatic inner layer. The electron periphery has twenty-petal adsorptive substances, forming a big magnetism radius. If two electrons with the same velocity and direction converge, their adsorptive substances have the same rotational frequency and phase. The magnetic force is $\sqrt{2}$ times the electrostatic force; the existence of adsorptive substance causes the magnetic force greater than the electrostatic force. The magnetic properties of iron, cobalt, and nickel can be explained through the regular dodecahedron electronic shell. The electron orbit converge from reverse direction can explain diamond. The behavior of the adsorptive substances is evolved into the magneton theory, and it can work well with thermodynamic. The free electrons do not have collision in high critical temperature superconductivity. In glass, the same ions will attract each other and can separately form an ion group of a similar type. The structure of the light is a helical line. The maximal shell numbers of the electronic shells are ten.

\section{REFERENCES}

[1] G. N. Lewis, Journal of the American Chemical Society, Vol. 38, 1916, pp. 762-785. http://dx.doi.org/10.1021/ja02261a002

[2] X. T. Lu, H. J. Wang and B. J. Yang, "Atomic Nucleus Physics,” Atomic Energy Press, Beijing, 1981, pp. 46-47.

[3] O. Echt, K. Sattler and E. Recknagel, Physical Review Letters, Vol. 47, 1981, p. 1121. http://dx.doi.org/10.1103/PhysRevLett.47.1121

[4] W. Miehle, O. Kandler, T. Leisner and O. J. Echt, Chemical Physics, Vol. 91, 1989, p. 5940

[5] P. W. Stephens and J. G. King, Physical Review Letters, Vol. 51, 1983, pp. 1538-1541.

http://dx.doi.org/10.1103/PhysRevLett.51.1538

[6] G. S. Tian, "The Origin of Metallic Ferromagnetism," In: 10000 Selected Problems in Sciences, Physics, Science Press, Beijing, 2009, pp. 73-80.

[7] H. Stöcker, "Taschenbuch der Physik," Verlag Harri, Deutsch, 1996.

[8] A. Chaiken, "Resistivity and specific Heat in Zero Applied Magnetic Field for a Typical Superconductor," 2005. http://en.wikipedia.org/wiki/File:Cvandrhovst.png

[9] Y. I. Frenkel, "Kinetic Theory of Liquids," Oxford University Press, Oxford, 1946.

[10] M. I. Ojovan, K. P. Travis and R. J. Hand, Journal of Physics: Condensed Matter, Vol. 19, 2007, Article ID: 
415107.

http://dx.doi.org/10.1088/0953-8984/19/41/415107

[11] R. H. Doremus, Journal of Applied Physics, Vol. 92, 2002, pp. 7619-7629. http://dx.doi.org/10.1063/1.1515132

[12] M. B. Volf, "Mathematical Approach to Glass," Elsevier,
Amsterdam, 1988.

[13] M. I. Ojovan and W. E. Lee, Journal of Applied Physics, Vol. 95, 2004, pp. 3803-3810.

http://dx.doi.org/10.1063/1.1647260 


\section{Appendix 1}

Cylinder and all five kinds of regular polyhedra (platonic solids).

\begin{tabular}{|c|c|c|c|c|c|c|}
\hline & \multirow{2}{*}{ Cylinder } & \multicolumn{5}{|c|}{ Regular polyhedra (platonic solids) } \\
\hline & & Tetrahedron & Hexahedron, cube & Icosahedron & Dodecahedron & Icosahedron \\
\hline \multicolumn{7}{|l|}{ Figure } \\
\hline Faces & 2 & 4 & 6 & 8 & 12 & 20 \\
\hline Edges & 2 & 6 & 12 & 12 & 30 & 30 \\
\hline Vertices & & 4 & 8 & 6 & 20 & 12 \\
\hline Edges each face & 1 & 3 & 4 & 3 & 5 & 3 \\
\hline Edges each vertex & & 3 & 3 & 4 & 3 & 5 \\
\hline
\end{tabular}

\section{Appendix 2}

$\begin{array}{lll}1 & \mathrm{H} & 1 \\ 2 & \mathrm{He} & 2 \\ 3 & \mathrm{Li} & 2,1 \\ 4 & \mathrm{Be} & 2,2 \\ 5 & \mathrm{~B} & 2,3 \\ 6 & \mathrm{C} & 2,4 \\ 7 & \mathrm{~N} & 2,5 \\ 8 & \mathrm{O} & 2,6 \\ 9 & \mathrm{~F} & 2,7 \\ 10 & \mathrm{Ne} & 2,8\end{array}$

21 Sc $2,8,8,3$

$22 \mathrm{Ti} 2,8,8,4$

$23 \mathrm{~V} 2,8,8,5$

$24 \mathrm{Cr} 2,8,8,4,2$

$25 \mathrm{Mn} \mathrm{2,8,8,4,3}$

$26 \mathrm{Fe} 2,8,12,3,1$

27 Co 2, 8, 12, 3, 2

$28 \mathrm{Ni} \quad 2,8,12,5,1$

$29 \mathrm{Cu} 2,8,12,6,1$

$30 \mathrm{Zn} \quad 2,8,12,6,2$

31 Ga 2, 8, 12, 6, 3

32 Ge 2, 8, 12, 6, 4

33 As 2, 8, 12, 6, 5

34 Se $2,8,12,6,6$

$35 \mathrm{Br} \quad 2,8,12,6,7$

$36 \mathrm{Kr} \quad 2,8,12,6,8$

$37 \mathrm{Rb} 2,8,12,6,8,1$

$38 \mathrm{Sr} \quad 2,8,12,6,8,2$

$39 \mathrm{Y} \quad 2,8,12,6,8,3$

$40 \mathrm{Zr} \quad 2,8,12,6,8,4$

$41 \mathrm{Nb} \quad 2,8,12,6,8,5$

42 Mo 2, 8, 12, 6, 8, 6

43 Tc 2, 8, 12, 6, 8, 4, 3

$44 \mathrm{Ru} 2,8,12,6,8,4,3,1$

45 Rh $2,8,12,6,8,4,3,2$

$\begin{array}{lll}11 & \mathrm{Na} & 2,8,1 \\ 12 & \mathrm{Mg} & 2,8,2 \\ 13 & \mathrm{Al} & 2,8,3 \\ 14 & \mathrm{Si} & 2,8,4 \\ 15 & \mathrm{P} & 2,8,5 \\ 16 & \mathrm{~S} & 2,8,6 \\ 17 & \mathrm{Cl} & 2,8,7 \\ 18 & \mathrm{Ar} & 2,8,8 \\ 19 & \mathrm{~K} & 2,8,8,1 \\ 20 & \mathrm{Ca} & 2,8,8,2\end{array}$

$2,8,8,2,1$

$2,8,8,3,1$

$2,8,8,4,1$

$2,8,8,3,3$

$2,8,8,5,2$

$2,8,12,2,2$

$2,8,12,4,2$

$2,8,12,4,3$

$2,8,12,8,1$

$2,8,12,8,2$

$2,8,12,8,3$

$2,8,12,8,4$

$2,8,12,8,5$

$2,8,12,6,8,3,1$

$2,8,12,6,8,4,1$

$2,8,12,6,8,4,2$

$2,8,12,6,8,5,2$

$2,8,12,6,8,4,2,2$

$2,8,12,6,8,6,3$ 
$97 \mathrm{Bk}$

$98 \mathrm{Cf}$

99 Es
$2,8,12,6,8,4,4,2$

$$
\begin{array}{ll}
2,8,12,6,12,6,1 & 2,8,12,6,12,4,2,1 \\
12,6,12,6,2 & 2,8,12,6,12,4,3,1 \\
2,8,12,6,12,6,3 & 2,8,12,6,12,6,2,1 \\
12,6,12,6,4 & 2,8,12,6,12,6,2,2 \\
12,6,12,6,5 & 2,8,12,6,12,6,3,2 \\
12,6,12,8,4 & 2,8,12,6,12,8,2,2 \\
2,8,12,6,12,6,7 & 2,8,12,6,12,6,5,2
\end{array}
$$

\author{
$2,8,12,6,12,4,3,1$ \\ $2,8,12,6,12,6,2,1$ \\ $2,8,12,6,12,6,2,2$ \\ $2,8,12,6,12,6,3,2$ \\ $2,8,12,6,12,8,2,2$
}$$
2,8,12,6,12,6,2
$$

$2,8,12,6,12,6,4$

$2,8,12,6,12,6,5$

$2,8,12,6,12,8,4$

$2,8,12,6,12,6,8$

$2,8,12,6,12,6,8,1$

$2,8,12,6,12,6,8,2$

$2,8,12,6,12,6,8,3$

$2,8,12,6,12,6,8,4$ $2,8,12,6,12,6,8,4,1$ $2,8,12,6,12,6,8,4,2$ $2,8,12,6,12,6,8,4,3$ $2,8,12,6,12,6,8,4,3,1$

$2,8,12,6,12,6,8,4,3,2$ $2,8,12,6,12,6,12,4,2$ $2,8,12,6,12,6,12,4,3$ $2,8,12,6,12,6,12,4,3,1$ $2,8,12,6,12,6,12,6,3$ $2,8,12,6,12,6,12,6,3,1$ $2,8,12,6,12,6,12,6,3,2$ $2,8,12,6,12,6,12,6,3,3$ $2,8,12,6,12,6,12,6,4,3$ $2,8,12,6,12,6,12,6,4,4$ $2,8,12,6,12,6,12,6,5,4$ $2,8,12,6,12,6,12,6,6,4$ $2,8,12,6,12,6,12,6,6,5$ $2,8,12,6,12,6,12,6,6,4,2$ $2,8,12,6,12,6,12,6,6,4,3$ $2,8,12,6,12,20,8,6,4$ $2,8,12,6,12,20,8,6,4,1$ $2,8,12,6,12,20,8,6,4,2$ $2,8,12,6,12,20,8,6,6,1$ $2,8,12,6,12,20,8,6,6,2$ $2,8,12,6,12,20,8,6,6,3$ $2,8,12,6,12,20,12,6,4,2$ $2,8,12,6,12,20,12,6,4,3$

$2,8,12,6,12,20,12,6,8$ $2,8,12,6,12,20,12,6,8,1$ $2,8,12,6,12,20,12,6,8,2$ $2,8,12,6,12,20,12,6,8,3$ $2,8,12,6,12,20,12,6,8,4$ $2,8,12,6,12,20,12,6,8,4,1$ $2,8,12,6,12,20,12,6,8,5,1$ $2,8,12,6,12,20,12,6,8,5,2$ $2,8,12,6,12,20,12,6,12,3,1$ $2,8,12,6,12,20,12,6,12,3,2$ $2,8,12,6,12,20,12,6,12,4,2$ $2,8,12,6,12,20,12,6,12,3,3$ $2,8,12,6,12,20,12,6,12,4,3$ $2,8,12,6,12,20,12,6,12,6,2$ $2,8,12,6,12,20,12,6,12,5,3$ $2,8,12,6,12,20,12,6,12,6,3$
$2,8,12,6,12,6,8,3,1$ $2,8,12,6,12,6,8,3,2$ $2,8,12,6,12,6,8,3,3$

$2,8,12,6,12,6,8,4,2,2$

$2,8,12,6,12,6,12,3,3$

$2,8,12,6,12,6,12,6,2$

$2,8,12,6,12,6,12,6,4,2$

$2,8,12,6,12,6,12,6,4,3,1$

$2,8,12,6,12,6,12,6,5,5$

$2,8,12,6,12,6,12,6,6,4,1$

$2,8,12,6,12,6,12,6,6,3,3$

$2,8,12,6,12,20,8,6,2,2$

$2,8,12,6,12,20,8,6,3,2$

$2,8,12,6,12,20,8,6,5,1$

$2,8,12,6,12,20,8,6,4,3$

$2,8,12,6,12,20,12,6,4$

$2,8,12,6,12,20,12,6,5$

$2,8,12,6,12,20,12,6,6,1$

$2,8,12,6,12,20,12,6,6,2$

$2,8,12,6,12,20,12,6,8,2,2$

$2,8,12,6,12,20,12,6,8,5$

$2,8,12,6,12,20,12,6,8,6$

$2,8,12,6,12,20,12,6,8,4,3$

$2,8,12,6,12,20,12,6,12,4$ 
$100 \mathrm{Fm} \quad 2,8,12,6,12,20,12,6,12,6,3,1$ $2,8,12,6,12,20,12,6,12,8,2$

$101 \mathrm{Md} \quad 2,8,12,6,12,20,12,6,12,8,3$

102 No $\quad 2,8,12,6,12,20,12,6,12,8,3,1$ $2,8,12,6,12,20,12,6,12,8,2,2$

$103 \mathrm{Lr} \quad 2,8,12,6,12,20,12,6,12,8,3,2$ 